\title{
LEVANTAMENTO DE QUANTITATIVOS DE PONTE: COMPARAÇÃO ENTRE O MÉTODO TRADICIONAL E BRIM
}

\author{
E. L. Machado ${ }^{1}$; S. Scheer ${ }^{2}$; A. P. L. Santos ${ }^{3}$ \\ 1,2,3 Universidade Federal do Paraná, Centro Politécnico, Curitiba/PR, CEP 81530-900 \\ eduarda.lauck@ufpr.br',scheer@ufpr.br',adrianapls@ufpr.br ${ }^{3}$
}

\begin{abstract}
Resumo: A determinação dos custos dos empreendimentos de construçáo civil é feita por meio do processo de orçamentação, cuja etapa inicial se trata do levantamento dos quantitativos de serviços e de insumos. Normalmente, o levantamento de quantitativos é realizado a partir da análise do projeto desenvolvido, das especificações técnicas e das plantas construtivas. Esse método pode, muitas vezes, apresentar-se falho, afetando, frequentemente, a tomada de decisão das empresas. Este artigo busca comparar o processo de levantamento de quantitativos de uma ponte pelo método tradicional e também com a utilização de software BIM (Building Information Modeling - Modelagem da Informação da Construção). A partir do BIM aplicado aos edifícios, surge o conceito de BrIM (do inglês, Bridge Information Modeling - Modelagem de Informaçôes de Pontes), aplicado a pontes. Para tanto, foram desenvolvidos três experimentos, comparando-se as diferenças entre o método convencional e os assistidos por software BIM. Observa-se que o método utilizando BrIM apresenta vantagens em relação ao método tradicional, no que se refere a precisão e a rapidez dos levantamentos.
\end{abstract}

Palavras-chave: Estimativa de custo, Levantamento de quantitativos, Modelagem da Informação da Construção (BIM), Modelagem da Informação de Ponte (BrIM).

Abstract: The determination of the cost of construction projects is made through the budget process, whose initial stage it is the quantity takeoff of services and inputs. Normally, the quantity takeoff is performed from project analysis developed, technical specifications and construction plans. This method can often present itself flawed affecting frequently the decision making of companies. This article aims to compare the quantity takeoff process of a bridge by the traditional method and with the use of BIM (Building Information Modeling) software. From BIM applied to buildings, the concept of BrIM (Bridge Information Modeling) emerges, applied to bridges. Therefore, three experiments were conducted, comparing the differences between the conventional method and methods assisted by BIM software. It is possible to note that the BrIM method presents advantages compared to the traditional method, as regards the accuracy and the speed of takeoff.

Keywords: Cost estimation, Quantity takeoff, Building Information Modelling (BIM), Bridge Information Modelling (BrIM). 


\section{Introduçáo}

A estimativa de custos, ou orçamentação, é uma etapa fundamental para a engenharia e influencia significativamente o planejamento, o projeto, a licitação, o gerenciamento de custos e até mesmo o gerenciamento da construção. São as estimativas que permitem que os proprietários e gestores possam avaliar os custos de viabilidade e controle de projetos de forma eficaz [1].

De acordo com [2], devido a orçamentação de obras se tratar de um procedimento quase totalmente manual, esta pode apresentar diversos equívocos, sendo que muitos deles ocorrem na etapa de levantamento de quantitativos, o que faz com que o erro se propague gradativamente em todo o orçamento. Sendo assim, um erro no levantamento de quantitativos pode refletir na avaliação do custo final de um empreendimento, afetando, assim, a tomada de decisão das empresas.

As pontes constituem um elo crítico na rede rodoviária e exigem um grande investimento de capital tanto para a construção quanto para manutenção. Essas estruturas, juntamente com os viadutos e túneis, são chamadas Obras de Arte Especiais (OAEs). Segundo dados de Custo Médio Gerencial do $\mathrm{DNIT}^{1}$, a construção de uma OAE de concreto armado tem custo médio de $\mathrm{R} \$ 4.080,20$ o metro quadrado de construção, considerando infraestrutura, mesoestrutura e superestrutura, sem os acessos e fundaçôes especiais, enquanto os projetos de uma OAE têm um custo médio de R \$100,00 o metro quadrado ${ }^{2}$, considerando os estudos, os levantamentos e as sondagens. Assim, um erro no levantamento de quantitativos de uma ponte pode afetar expressivamente o orçamento de sua construção.

No processo tradicional de execução de um projeto de construção civil, em que cada conjunto de informaçôes é agregado ao processo, podem ocorrer falhas na fase de planejamento e, principalmente, de levantamento de custos do empreendimento, decorrentes da dificuldade de coordenar e alinhar todas as informaçóes inerentes ao processo [3].

De acordo com a referência [4] o levantamento de quantitativos é uma das tarefas-chave no processo de construção, pois é a base para várias outras tarefas, os elementos de construção são medidos e as quantidades são entáo utilizadas para estimar o custo e a carga de trabalho relevante. Ainda segundo os autores, o levantamento de quantitativos nos estágios iniciais fornece a base para uma estimativa preliminar de custos para o projeto. Já na fase de orçamentação é utilizado para auxiliar na estimativa do custo e duração das atividades de construção do projeto, antes da fase de construção é usado para prever e planejar as atividades de construção; e durante a fase de construção é utilizado para o controle de custos do projeto.

Segundo a referência [5], a ausência de padronização no levantamento dos quantitativos em um projeto pode ser apontada como uma das fontes de imprecisão no custo final do orçamento, da

\footnotetext{
${ }^{1}$ Dados de setembro de 2016. Disponível em http://www.dnit.gov.br/custos-e-pagamentos/copy_of_custo-medio-gerencial. ${ }^{2}$ Dados de janeiro de 2014. Disponível em http://www.dnit.gov.br/custos-e-pagamentos/copy_of_custo-medio-gerencial. 
mesma maneira que o desconhecimento sobre a relação entre o coeficiente de consumo da composição de custo e seu respectivo critério de medição.

Conforme a referência [6], o levantamento de quantitativos 2D faz parte do processo tradicional de orçamentação, que é baseado nos projetos impressos e executado por disciplinas. O responsável por este levantamento de quantitativos mede cada elemento do projeto, identificando-o por meio de um código ou pelo seu ambiente.

A referência [4] destaca ainda, que uma vez que cada elemento é medido individualmente, essa medição pode conter inferências e interpretaçôes pessoais do projeto, ou seja, mesmo sendo baseados nas mesmas especificaçôes, dois levantamentos de quantitativos, executados por indivíduos diferentes, podem apresentar resultados diferenciados.

Tratando-se de elaboração de orçamentos, etapa em que se tem prazos mais enxutos, muitas vezes não há tempo hábil para a solicitação de materiais de acordo com o planejamento desejado para a obra. É, portanto, nesta etapa que a extração de quantitativos com uso da modelagem de informaçóes da construção (Building Information Modelling ou BIM) objetiva atuar, pois uma vez que o modelo detém todas as informaçóes necessárias de projeto, obtém-se facilmente o levantamento de quantitativo, e assim espera-se que haja menos desvios nos cálculos, melhor rastreabilidade da informação e maior flexibilidade na obtenção dos dados [6].

A referência [7] define o BIM como "um conjunto de tecnologias, processos e políticas que permitem que várias partes interessadas possam projetar, construir e operar uma instalação de forma colaborativa”. O BIM é um método que aborda o projeto de construção e toda documentação do mesmo, considerando todo o ciclo de vida do empreendimento, incluindo informaçóes relacionadas à concepção, simulação e operacionalização através da utilização de diferentes ferramentas integradas [8].

$\mathrm{Na}$ literatura é possível encontrar vários trabalhos que apresentam o BIM para o levantamento de quantitativos $[2,4,6,9,10]$. Destaca-se assim, o grande potencial da tecnologia BIM no levantamento de quantitativos de obras.

A partir do BIM aplicado aos edifícios, surge o conceito de BrIM (do inglês, Bridge Information Modeling - Modelagem de Informaçôes de Pontes), aplicado a pontes. A referência [11] aponta que o BrIM tornou-se uma ferramenta amplamente eficaz na indústria de engenharia e construção de pontes, visto que não se trata apenas uma representação geométrica de pontes, mas uma representação inteligente de pontes, pois contém todas as informaçôes necessárias sobre pontes ao longo de seu ciclo de vida.

A referência [11] define o BrIM como um modelo 3D com componentes inteligentes. O modelo 3D da ponte pode ser usado para: atualizaçáo de desenhos, levantamento de quantitativos e lista de materiais; enquanto o modelo $4 \mathrm{D}$ pode conectar elementos de modelo a cronogramas de tempo, e o modelo 5D, integra a estimativa de custo com os componentes do modelo. 
Desta forma, o BrIM tem grande efeito na melhoria das três principais preocupaçóes dos stakeholders de pontes, que são qualidade, cronograma e custo, e é necessária para pontes, pois cria consistência na informação em diferentes fases do projeto à manutenção [12].

$\mathrm{Na}$ literatura encontram-se pesquisas focadas diretamente sobre a integração das etapas de construção de uma ponte, através da concepção e construção, manutenção e operação para a estrutura $[9,13-16]$.

Deste modo, este artigo tem como objetivo apresentar uma comparação entre os levantamentos de quantitativos para a construção de uma ponte de concreto armado, utilizando o método convencional e softwares de plataforma BIM. Para tanto serão realizados dois experimentos comparando-se as diferenças entre os levantamentos realizados utilizando o software AutoCAD e dois softwares de plataforma BIM, o SketchUp, que um software de modelagem 3D, e o Revit, uma ferramenta BIM multidisciplinar que permite simular uma obra no seu ambiente gráfico.

\section{Método}

A primeira etapa desta pesquisa consistiu numa revisão bibliográfica apresentada na seção de introdução. Esta revisão, do tipo narrativa, não se utilizou critérios explícitos e sistemáticos para a busca e análise crítica da literatura, uma vez que não tinha como objetivo esgotar as fontes de informaçôes [17].

O método de pesquisa adotado para este artigo foi a pesquisa experimental, que consiste em determinar um objeto de estudo, selecionar as variáveis que seriam capazes de influenciá-lo e definir as formas de controle e de observaçáo dos efeitos que a variável produz no objeto. Cabe destacar que este método de pesquisa não precisa necessariamente ser realizado em laboratório [18].

A ponte para a qual foi realizada o levantamento de quantitativos é uma estrutura já existente, situada no estado do Paraná e é composta por uma plataforma de seção caixão, com 67,5 m de comprimento e $12,5 \mathrm{~m}$ de largura. A plataforma tem uma altura toral de 2,62 $\mathrm{m}$ e está apoiada sobre 3 pares de pilares de seção circular com $8 \mathrm{~m}$ de comprimento e raio igual à $0,4 \mathrm{~m}$. Na base de cada par de pilares existe uma viga com $0,5 \mathrm{~m}$ de base, $0,8 \mathrm{~m}$ de altura e 3,83 $\mathrm{m}$ de comprimento. A fundação é formada por sapatas circulares cônicas (de base maior com 1,6m de raio e base menor de $0,5 \mathrm{~m}$ de raio), com uma base cilíndrica de 1,6 m de raio e 0,3 m de altura. Os planos $2 \mathrm{D}$ e a modelagem da ponte nos softwares SketchUp e Revit foram feitos a partir de material fotográfico procedentes de relatórios de inspeção.

Nos experimentos buscou-se obter o levantamento de quantitativos de: volume de concreto da estrutura, área a ser coberta com asfalto, área a ser coberta de tinta azul (área externa da plataforma) e com tinta vermelha (área externa dos pilares). 
Foram propostos três diferentes experimentos, em que o primeiro tem como objetivo obter os quantitativos de materiais pelo método tradicional, o segundo objetiva levantar esses quantitativos utilizando um software com plataforma BIM mais simples, o SketchUp, e o terceiro utilizando outra plataforma BIM, o Revit.

O experimento pelo método tradicional utilizou como base os planos $2 \mathrm{D}$ da ponte, a partir dos quais se realiza de forma manual o levantamento de materiais utilizados na estrutura da ponte. Para tanto foi utilizado o software AutoCAD 2018, em versão educacional. Nesta etapa os materiais foram medidos diretamente no software (Figura 1), com o uso de suas ferramentas, e organizados em planilhas.

Para o segundo experimento, com plataforma BIM, foi feita a modelagem da ponte no SketchUp Make 2017 (Figura 2), de forma que pudessem ser obtidos os quantitativos de materiais, por meio das dimensôes dos sólidos gerados no modelo.

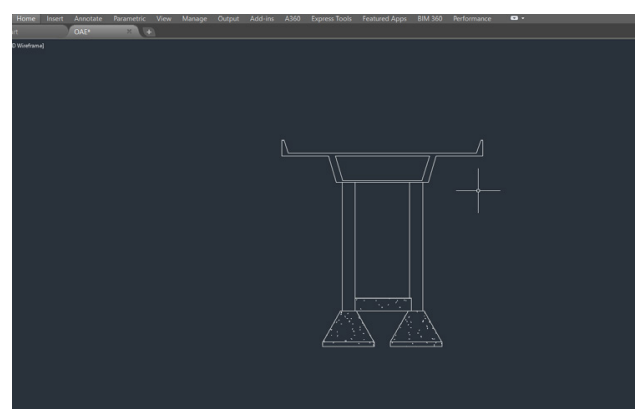

Figura 1 - Planos 2D da ponte no AutoCAD.

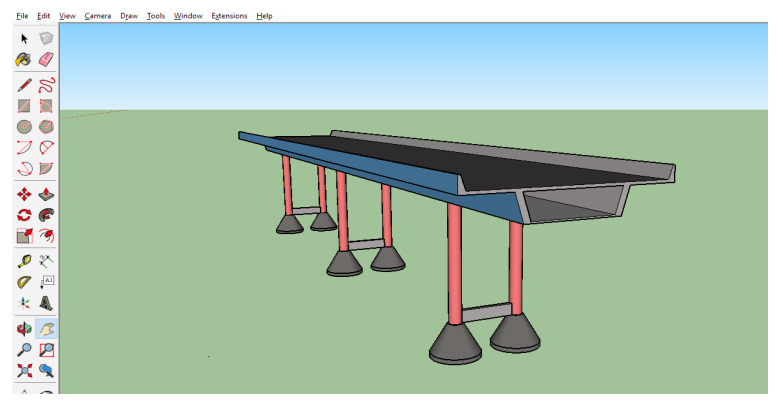

Figura 2 - Modelagem da ponte no SketchUp.

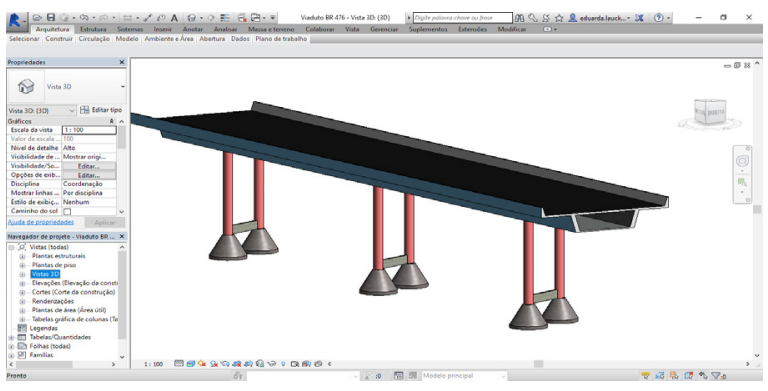

Figura 3 - Modelagem da ponte no Revit.

O terceiro experimento utilizou a modelagem da ponte no software Revit 2017, em sua versão educacional (Figura 3), como plataforma BIM, do qual se obtém, facilmente, o quantitativo de materiais. A partir da caracterização paramétrica dos objetos da ponte foi possível realizar a elaboração de tabelas de quantitativos. Este software permite a quantificação dos materiais com base nos componentes criados para representá-los, isto é, a criação de tabelas se baseia na quantificação do material escolhido para representar o objeto. 


\section{Resultados}

Ainda que a ponte seja uma grande estrutura, sua modelagem está constituída por uma pequena variedade de materiais. Neste levantamento foi considerada apenas a quantidade de concreto presente na estrutura, concreto asfáltico, e tintas.

No primeiro experimento, com o uso do AutoCAD, foi realizado o levantamento diretamente do arquivo em plataforma CAD 2D. As medidas dos elementos da ponte foram registradas em uma planilha eletrônica, onde foram calculados os quantitativos de cada parte da estrutura.

No segundo experimento, que corresponde à modelagem da estrutura no SketchUp, os valores do volume de concreto bem como os valores das áreas de asfalto e de superfície a ser coberta por tinta não foram obtidos automaticamente. Uma vez selecionado um determinado elemento na modelagem, o software informa seu volume e sua área. Apenas os volumes da plataforma e da fundaçáo tiveram que ser calculados manualmente, devido à complexidade de suas geometrias. Por se tratar de uma estrutura constituída por uma pequena variedade de partes, o levantamento das quantidades não foi complicado, apesar de não ser automático.

Vale ressaltar que dependendo da geometria dos elementos da ponte, o levantamento de quantitativos no SketchUp pode ser realizado por meio de dois plugins, o Bimbon e o Cut List. Estes plugings permitem a quantificação dos materiais com base nos materiais e componentes utilizados para representá-los. Assim, se os elementos estruturais da ponte forem pré-fabricados, estes podem ser acrescentados à modelagem utilizando o plugin Bimbon, cuja lista de itens e orçamento é gerada automaticamente. Caso a geometria seja de seçóes retangulares, o Cut List é capaz de realizar o levantamento de quantitativos. Devido às particularidades da ponte em questão, nenhum destes plugins poderia realizar o levantamento de quantitativos adequado.

As quantidades obtidas a partir dos levantamentos dos três experimentos podem ser verificadas na Tabela 1 .

Tabela 1 - Levantamento de quantitativo da ponte.

\begin{tabular}{|c|c|c|c|c|}
\hline Material & Elemento & $\begin{array}{c}\text { Levantamento } \\
\text { tradicional }\end{array}$ & $\begin{array}{c}\text { Levantamento } \\
\text { SketchUp }\end{array}$ & $\begin{array}{c}\text { Levantamento } \\
\text { Revit }\end{array}$ \\
\hline \multirow{5}{*}{ Concreto $\left(\mathrm{m}^{3}\right)$} & Fundação & 56,69 & 56,69 & 57,00 \\
\hline & Pilares & 24,13 & 24,13 & 24,12 \\
\hline & Plataforma & 306,11 & 306,11 & 306,11 \\
\hline & Vigas & 1,31 & 1,83 & 1,83 \\
\hline & Total & 388,24 & 388,76 & 389,06 \\
\hline Asfalto $\left(\mathrm{m}^{2}\right)$ & Plataforma & 789,75 & 789,75 & 789,75 \\
\hline Tinta Azul $\left(\mathrm{m}^{2}\right)$ & Plataforma & 1139,40 & 1135,62 & 1136,99 \\
\hline Tinta Vermelha $\left(\mathrm{m}^{2}\right)$ & Pilares & 120,64 & 120,29 & 120,43 \\
\hline
\end{tabular}


Os quantitativos obtidos pelo Revit apresentam uma pequena diferença em relação aos obtidos pelo método tradicional e pelo SketchUp. Diferenças entre os levantamentos obtidos pelo método tradicional e os softwares de tecnologia BIM são observadas no volume total de concreto da estrutura, em que o levantamento de quantitativo manual é o menor valor obtido entre os três métodos $\left(388,24 \mathrm{~m}^{3}\right)$, e nas áreas a serem cobertas com tinta azul e vermelha, o método manual apresentou os maiores valores dentre os três $\left(1139,40 \mathrm{~m}^{2}\right.$ e 120,64 $\mathrm{m}^{2}$ respectivamente). Esse resultado já era esperado, visto que, os quantitativos foram extraídos por métodos diferentes, sendo que parte desses levantamentos foram obtidos de forma total ou parcialmente manual e sujeito a erros humanos.

Embora a diferença entre os valores seja muito pequena, em uma estrutura de maior porte, com uma maior quantidade de partes e mais volumosa, tal diferença poderia ser de fato significativa. A diferença observada nos experimentos não é apenas quantitativa. Vale enfatizar também que cada um dos métodos possui suas vantagens e desvantagens.

Apesar de predisposto a erros, o levantamento dos quantitativos pelo método manual proporciona maior facilidade de uso, uma vez que não apresenta necessidade de conhecimento de ferramentas avançadas de tecnologia da informação, diferentemente da plataforma BIM, que exige certo conhecimento por parte do projetista. Entretanto o processo manual é muito mais demorado que àqueles auxiliados por tecnologia BIM.

Por outro lado, o levantamento de quantitativos através de plataforma BIM propicia maior precisão dos materiais quantificados, já que os quantitativos extraídos são exatos, precisamente calculados pelas dimensôes do modelo BIM. Utilizando a plataforma consegue-se também maior rapidez do levantamento, pois as tabelas de quantitativos fornecidas pelos softwares são obtidas quase instantaneamente, enquanto pelo método manual, o levantamento é um processo demorado, que despende um nível elevado de esforço, e que, ainda assim, é passível de erros. Além disso, no caso de uma alteração de projeto, esta é automaticamente refletida nos levantamentos de quantitativos, com o uso de uma plataforma BIM, o que não ocorre no método tradicional.

\section{Conclusóes}

Este trabalho teve por objetivo comparar o levantamento de quantitativos de uma ponte considerando o método tradicional e também a utilização de tecnologia BIM. Para tanto, realizou-se o levantamento do volume de concreto, área de asfalto e de tinta numa ponte, pelos métodos propostos, e obteve-se como resultado uma pequena diferença, entre os valores de quantitativos extraídos, o que pode ser atribuído ao fato de que o levantamento pelo método tradicional está sujeito a erro humano inerente ao processo.

Contudo, no experimento utilizando um software de tecnologia BIM, o Revit, observou-se que os quantitativos decorrentes diretos da modelagem, são fornecidos de maneira precisa e rápida. Assim, esta tecnologia BIM se mostra muito eficiente na etapa do levantamento de quantitativos. 
Como sugestão para trabalhos futuros, propóem-se a realização de estudos sobre os custos associados ao uso dos métodos tradicional e BrIM.

\section{Agradecimentos}

Ao colega Claudio Cesar Wajdowicz pelo auxílio com a modelagem da ponte, no software Revit.

\section{Referências}

[1] CHENG, M. Y.; TSAI, H. C.; HSIEH, W. S. Web-based conceptual cost estimates for construction projects using Evolutionary Fuzzy Neural Inference Model. Automation in Construction, v. 18, n. 2, p. 164-172, 2008.

[2] SANTOS, A. P. L.; ANTUNES, C. E.; BALBINOT, G. B. Levantamento de Quantitativos de obras: comparação entre o método tradicional e experimentos em tecnologia BIM. Iberoamerican Journal of Industrial Engineering, v. 6, n. 12, p. 134-155, 2014.

[3] SAKAMORI, M. M.; SCHEER, S. Processo de extração de quantitativos de um modelo BIM 5D. In: Congresso Técnico Científico da Engenharia e da Agronomia, CONTECC 2016.

[4] MONTEIRO, A.; POÇAS MARTINS, J. A survey on modeling guidelines for quantity takeoff-oriented BIMbased design. Automation in Construction, v. 35, p. 238-253, 2013.

[5] MARCHIORI, F. F. Desenvolvimento de um método para elaboraçáo de redes de composiçáo de custos para orçamentaçáo de obras de edificaçóes. 2009. 238f. Tese (Doutorado em Engenharia) - Escola Politécnica da Universidade de São Paulo, São Paulo.

[6] MELHADO, S.; PINTO, A. C. Benefícios e desafios da utilização do BIM para extração de quantitativos. In SIBRAGEC ELAGEC 2015, São Carlos. 2015.

[7] SUCCAR, B. Building Information Modeling: Organisational Implementation \& Macro Adoption. International Forum on BIM. 2016.

[8] JALAEI, F; JRADE, A; NASSIRI, M. Integrating Decision Support System (DSS) and Building Information Modeling (BIM) to Optimize the Selection of Sustainable Building Components. Journal of Information Technology in Construction (ITcon), v. 20, n. 25, p. 399-420, 2015.

[9] CARDONA, J. S. G.; CABRERA, A. G. Uso de la metodología BrIM (Bridge Information Modeling) como herramienta para la planificación de la construcción de un puente de concreto en Colombia. Ciencia e Ingeniería Neogranadina, v. 24, n. 2, p. 145-156. 2014.

[10] COSTA, J. M. C.; SERRA, S. M. B. Comparação de processos de levantamento de quantitativos: tradicional e BIM. In ENCONTRO NACIONAL DE TECNOLOGIA DO AMBIENTE CONSTRUÍDO (ENTAC). n. 1, p. 2862-2871, 2014

[11] MARZOUK, M.; HISHAM, M. Bridge information modeling in sustainable bridge management. In: ICSDC 2011: Integrating Sustainability Practices in the Construction Industry. 2012. p. 457-466.

[12] MARZOUK, M; HISHAM, M. Implementing earned value management using bridge information modeling. KSCE Journal of Civil Engineering, v. 18, n. 5, p. 1302-1313, 2014.

[13] HALFAWY, F. C.; HADIPRIONO, F. C.; DUANE, J.; LAREW. Development of model based systems for integrated design of highway bridges. In: Institute for Research in Construction. International Conference on Civil, Structural and Environmental Engineering Computing, Roma, Italia. 2005.

[14] KIVIMAKI, T.; HEIKKILA, R. Bridge Information Modelling (BrIM) and Model Utilization at Worksites in Finland. In: 27th International Symposium on Automation and Robotics in Construction (ISARC 2010). 2010. 
[15] SHIN, H. M.; LEE, H. M.; OH, S. J.; CHEN, J. H. Analysis and design of reinforced concrete bridge column based on BIM. Procedia Engineering, v. 14, p. 2160-2163, 2011.

[16] SHIM, C; YUN, N. R.; SONG, H. H. Application of 3D bridge information modeling to design and construction of bridges. Procedia Engineering, v. 14, p. 95-99, 2011.

[17] INSTITUTO DE PSICOLOGIA - USP. BIBLIOTECA DANTE MOREIRA LEITE. Disponível em: <http:// www.ip.usp.br/portal/images/biblioteca/revisao.pdf>.

[18] GIL, A. C. Como elaborar projetos de pesquisa. 4. ed. São Paulo: Atlas, 2002. 